\title{
ON CATEGORIES WITH EFFECTIVE UNIONS
}

\author{
MICHAEL BARR
}

\begin{abstract}
We study an exactness condition that allows us to treat many of the familiar constructions in abelian categories and toposes in parallel. Examples of such constructions include Grothendieck's theorem on the existence of injective cogenerators, the exactness of right exact functors and torsion theories/topologies.
\end{abstract}

\section{Introduction}

One of the most surprising results in the early days of category theory - the one that demonstrated that there was power in the notion - was Grothendieck's theorem that what we now call a Grothendieck abelian category has an injective cogenerator, [Grothendieck, 1957]. The hypotheses of that theorem include two fundamental ideas. The first is that of an abelian category [Buchsbaum, 1956] that people had been zeroing in on since Mac Lane's influential paper, [Mac Lane, 1950]. The second was the infinite exactness condition that characterizes a Grothendieck category and, for abelian categories, goes under the name AB5.

It is easy to divorce the latter condition from that of additivity. One simply supposes that finite limits commute with filtered colimits. And indeed, this has been a very important concept in equational categories and many other places. Not so successful have been attempts to translate the finite exactness conditions into a non-additive setting. For example, it has been difficult to find a finite exactness condition that, together with exactness of filtered colimits, guarantees the existence of enough injectives. Of course, toposes have enough injectives, but the conditions defining toposes are much more than exactness conditions. (By an exactness condition, I mean any condition that says a limit and a colimit commute.)

Another interesting property that abelian categories share with toposes is that a functor between abelian categories is left exact if it preserve finite products and monos and is right exact.

One would hope that results like these could be shown to follow from well chosen exactness conditions. Unfortunately, insufficiently many exactness conditions are known that would permit one to prove the results above. In fact, the principal exactness property

Originally published in Lecture Notes in Mathematics 1348, the proceedings of a conference held in Louvain-la-Neuve in 1987.

The author of this paper was supported by the Ministère de l'Education du Québec through FCAR grants to the Groupe Interuniversitaire en Etudes Catégoriques and by an individual operating grant from the National Science and Engineering Research Council.

2000 Mathematics Subject Classification: 18A20, 18E40, 18 G05.

Key words and phrases: effective unions, injective cogenerators, torsion theories.

(c) Springer-Verlag, Berlin, Heidelberg, New York, 1988. 
of toposes - the universal effective sums - is not enjoyed by abelian categories, while they in turn have exactness properties not possessed by toposes.

The main purpose of this paper is to explore an exactness condition which is satisfied by both abelian categories and toposes and which is strong enough to prove (under relatively straightforward additional conditions) both the theorem on the existence of injective envelopes and the left exactness of certain functors. For example, this gives a new proof of the left exactness of the associated sheaf functor as a special case of reflectors for certain kinds of topologies on categories.

We apply these results to topologies, also known as torsion theories, by proving that, under appropriate additional conditions, the sheaf, that is torsion-free divisible, reflector exists and is exact. Although it was previously known that topologies were a non-abelian analog of torsion theories (see [Barr, 1973], the analogy has rarely, if ever, been fully exploited.

\section{Effective unions}

Definition. A category is said to have effective unions if in any pullback diagram

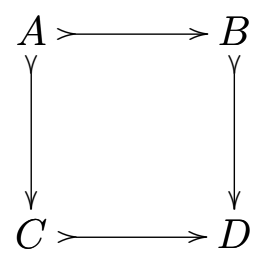

if all the indicated arrows are regular monomorphisms, then the pushout $B+{ }_{A} C$ exists and the arrow $B+{ }_{A} C \longrightarrow D$ is also a regular mono. This condition is satisfied in abelian categories and toposes. For the former, it follows from the fact that when $(*)$ is a pullback, then

$$
0 \longrightarrow A \longrightarrow B \oplus C \longrightarrow D
$$

is exact and then that $(B \oplus C) / A \longrightarrow D$. For toposes, it is proved in [Johnstone, 1977], p. 41. It is readily inferred from the following:

2.1. Proposition. A regular category with finite limits and colimits in which finite sums are disjoint and universal and every mono is regular has effective unions.

Proof. Let $E$ be the union in the subobject lattice of $D$ of the subobjects $B$ and $C$. Then

$$
(B+C) \times_{E}(B+C) \Longrightarrow B+C \longrightarrow E
$$

is a kernel pair/coequalizer diagram. Since $E \subseteq D$, the pullback over $E$ is the same as that over $D$. Because of the universality of sums,

$$
(B+C) \times \times_{D}(B+C) \cong B \times{ }_{D} B+B \times{ }_{D} C+C \times \times_{D} B+C+{ }_{D} C \circ
$$


But with $B \longrightarrow D$ and $C \longrightarrow D$, the first and last terms add up to the diagonal and the two middle terms are simply the symmetric versions of each other. In other words, $B \times{ }_{D} C=C \times{ }_{D} B=B \cap C$. Thus,

$$
B \cap C \Longrightarrow B+C \longrightarrow E
$$

is a coequalizer which implies that

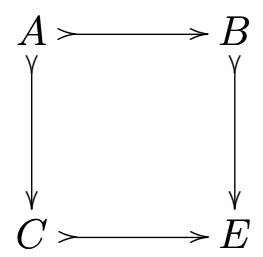

is a pushout.

2.2. The condition is inherited by slices, coslices, products and disjoint unions of categories and the formation of functor categories. It is also satisfied by the category of sheaves for a topology. (For the definition of topology, see Section 5 below.) The reason is that in all these cases connected finite limits and connected finite colimits are created in the original category.

Other examples include the category of compact Hausdorff spaces as well as by various full subcategories, like Stone spaces. The reason is that subspaces in those categories are closed and a function on a union of closed subspaces is continuous if its restriction to the subspaces is. John Isbell has observed that this condition will be satisfied in any variety or even quasi-variety whose theory includes no non-trivial finitary operation. The reason is that in those cases, the underlying set functor creates finite limits and colimits. Nevertheless, effective unions remain a relatively rare property.

In various parts of this paper, there will other exactness conditions required. In order to avoid having to impose different and quite technical conditions, we will simplify the presentation by supposing our categories to be biregular by which we mean that every morphism factors as a regular epimorphism followed by a regular monomorphism and that these factorizations are preserved by pushouts and pullbacks.

\section{Injectives}

Definition. Let $\mathscr{G}$ be a full subcategory of $\mathscr{C}$. We say that an object $Q$ is $\mathscr{G}$-injective if whenever $H \longrightarrow G$ is a regular monic in $\mathscr{C}$ between objects of $\mathscr{G}$, then $\operatorname{Hom}(G, Q)$ $\longrightarrow \operatorname{Hom}(H, Q)$ is surjective. An object is called injective if it is $\mathscr{C}$-injective.

3.1. Lemma. Suppose the category $\mathscr{C}$ and full subcategory $\mathscr{G}$ satisfy the following conditions:

(a) $\mathscr{C}$ has finite limits and exact filtered colimits; 
(b) $\mathscr{C}$ has pushouts of regular monos and they are regular monos;

(c) $\mathscr{G}$ is small;

(d) $\mathscr{C}$ is well-powered with respect to regular subobjects.

Then each object of $\mathscr{C}$ can be embedded by a regular mono into a $\mathscr{G}$-injective.

Proof. Fix an object $C$ of $\mathscr{C}$. Begin by well-ordering the set of all diagrams

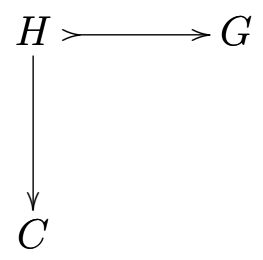

in which $H \longrightarrow G$ is a regular monomorphism between objects of $\mathscr{G}$. We will take this to mean that a one-one correspondence has been chosen between a set of ordinals $\alpha\langle\lambda$ and diagrams

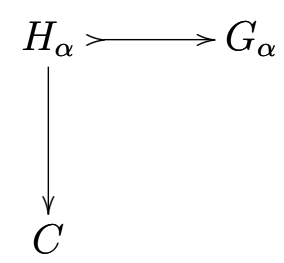

We will construct an ordinal sequence of objects $C_{\alpha}$ and regular monos $m_{\alpha \beta}: C_{\beta} \longrightarrow C_{\alpha}$, for $\beta \leq \alpha$, subject to the usual commutativity conditions as follows. Begin by letting $C_{0}=C$. If $\alpha$ is a limit ordinal and $C_{\beta}$ has been constructed for all $\beta\left\langle\alpha\right.$, we let $C_{\alpha}=$ $\operatorname{colim}_{\beta\langle\alpha} C_{\beta}$. For $\beta \leq \alpha$, we define $m_{\alpha \beta}$ to be the transition map to the colimit. We leave it to the reader to show, using the exactness of the filtered colimits, that it is a regular monic. We define $C_{\alpha+1}$ so that

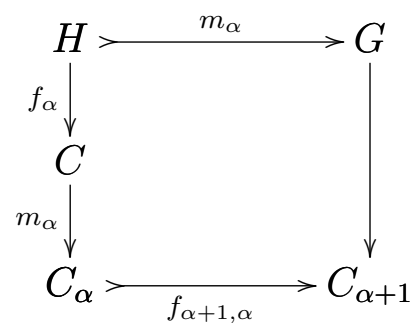

is a pushout and $m_{\alpha+1, \beta}=f_{\alpha+1, \alpha}{ }^{\circ} m_{\alpha \beta}$. We let $Q_{1}(C)=C_{\lambda}$. It is evident that each diagram

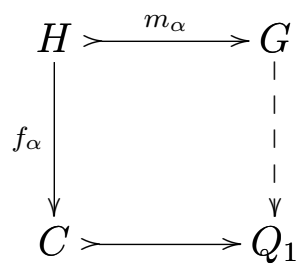


can be completed as shown. Next we choose a regular cardinal $\mu$ with the property that each object of $\mathscr{G}$ has a regular subobject lattice of cardinality less than $\mu$. Then we define an ordinal sequence $Q_{\alpha}(C)$ and regular monics $n_{\alpha \beta}: Q_{\beta}(C) \longrightarrow Q_{\alpha}(C)$ for $\beta \leq \alpha\langle\mu$ by letting $Q_{\alpha}(C)=\operatorname{colim}_{\beta\langle\alpha} Q_{\beta}(C)$ when $\alpha$ is a limit ordinal and $Q_{\alpha+1}(C)=Q_{1}\left(Q_{\alpha}(C)\right.$ ). Finally, $Q(C)=Q_{\mu}(C)$. I claim that any arrow $f: G \longrightarrow Q(C)$ with $G$ an object of $\mathscr{G}$ factors through $Q_{\alpha}(C)$ for some $\alpha\langle\mu$. In fact, filtered colimits commute with finite limits so if we let $G_{\alpha}=f^{-1}\left(Q_{\alpha}(C)\right)$, we have that colim $G_{\alpha}=G$. However, this colimit is taken over a set of regular subobjects of $G$ of cardinality larger than that of the whole subobject lattice of $G$. If we eliminate repetitions, we get $G$ as the colimit of a set of subobjects $G_{\alpha}$ where now the index set runs over a set of ordinals smaller than $\mu$. If we denote this index set by $I$, the fact that $\mu$ is regular implies that $\sup \{\alpha \| \alpha \in I\}=\nu\langle\mu$. But $\operatorname{colim}_{\alpha \in I} G_{\alpha}=G$ which means that $f$ factors through $Q_{\nu}(C)$. Now if we have a diagram

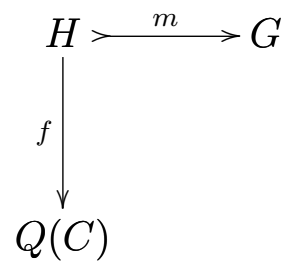

it can be factored and then filled in as indicated

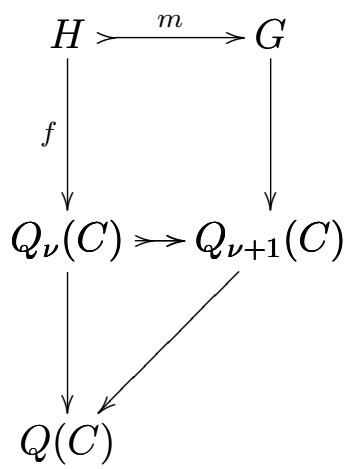

3.2. Proposition. Suppose that in addition to the hypotheses of 3.1, $\mathscr{C}$ has effective unions and cokernel pairs and $\mathscr{G}$ is closed under epimorphic images and regular subobjects and includes a set of strong generators. Then a $\mathscr{G}$-injective is injective.

This argument is adapted from the one found in [Grothendieck, 1957] and really goes back to Baer's proof that a divisible abelian group is injective.

Proof. Let $Q$ be a $\mathscr{G}$-injective and consider a diagram

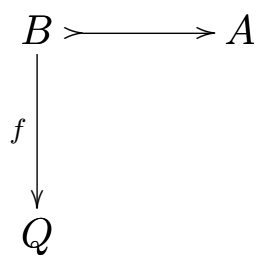


in which $B \longrightarrow A$ is a proper regular mono. There is an object $G$ of $\mathscr{G}$ and an arrow $G$ $\longrightarrow A$ that does not factor through $B$. Under the hypothesis 3.1(b) and the existence of cokernel pairs every arrow factors as an epi followed by a regular mono and $\mathscr{G}$ is closed under quotients, we can suppose that $G \longrightarrow A$ is a regular mono. Then $H=G \times_{A} B$ is a proper regular subobject of $G$. We have the diagram

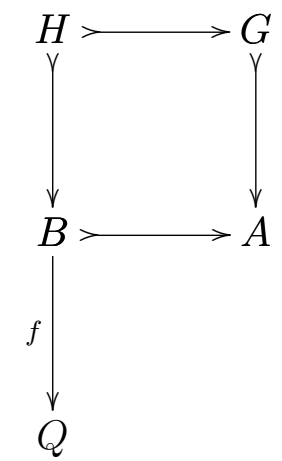

The pushout $G+_{H} B$ is by hypothesis a regular subobject of $A$ that properly contains $B$ and the mapping property of the pushout implies that the map extends to it. This shows that $f$ can be extended a little and a standard Zorn's lemma argument (using the exactness of filtered colimits) takes us the rest of the way.

3.3. TheOREM. Suppose the category $\mathscr{C}$ is a category and $\mathscr{G}$ a full subcategory that satisfy the following conditions:

(a) $\mathscr{C}$ is regular and coregular and all monos are regular;

(b) $\mathscr{C}$ has exact filtered colimits;

(c) pushouts of regular monos are regular monos;

(d) $\mathscr{C}$ has effective unions;

(e) $\mathscr{C}$ is co-well-powered;

(f) $\mathscr{G}$ includes a set of strong generators.

Then $\mathscr{C}$ has enough injectives.

Proof. Since $\mathscr{C}$ has a set of generators, it is also well-powered and so we can suppose that $\mathscr{G}$ is closed under subobjects and regular quotient objects. Then the hypotheses of 3.1 and 3.2 are satisfied and the conclusion follows. 
3.4. InjeCtive COGENERAtors. We have shown that there are enough injectives. We wish to show that Grothendieck's argument about the existence of injective cogenerators can also be adapted to this setting. We begin by closing up the set of generators under finite sums and also under quotients. Then every object is a monomorphic filtered colimit of generators. For each generator $G$, let $Q(G)$ denote an injective container of $G$. Let $\mathscr{Q}$ denote the set of the $Q(G)$.

3.5. TheOREM. A locally finitely presentable category in which all epis are regular and which has exact filtered colimits, a small generating subcategory, and sufficient injectives has a small injective cogenerating family.

PROOF. We can suppose that the generating subcategory is small and closed under finite sums, finite products, subobjects and quotient objects. Let $\mathscr{C}$ and $\mathscr{G}$ denote the category and the small generating subcategory, respectively.

Given a proper epimorphism $B \longrightarrow A$, let $K \longrightarrow B$ be the kernel pair. Write $B=\operatorname{colim} G_{i}$ a monomorphic filtered colimit with the $G_{i}$ objects of $\mathscr{G}$. Since $K=B \times{ }_{A} B$, it follows that $K$ is the colimit of the diagram

$$
\left(\operatorname{colim} G_{i}\right) \times_{A}\left(\operatorname{colim} G_{i}\right) \cong \operatorname{colim}\left(G_{i} \times_{A} G_{i}\right)
$$

Since the two arrows from $K$ to $B$ are distinct (otherwise $B \longrightarrow A$ is not a proper epi), it follows that for some index $i$ the two composite arrows

$$
G_{i} \times{ }_{A} G_{i} \longrightarrow B \times B \longrightarrow B \longrightarrow A
$$

are distinct. Now consider the serially commutative diagram

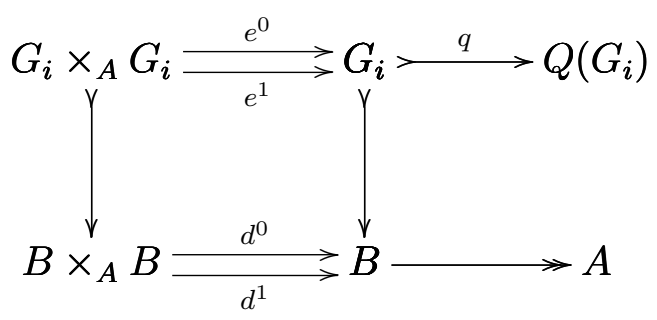

The injectivity forces the existence of an arrow $g: B \longrightarrow Q\left(G_{i}\right)$ making the triangle commutative. Since $e^{0} \neq e^{1}$ and $q$ is mono, it follows that $q \circ e^{0} \neq e^{1}$ hence $g \circ d^{0} \neq d^{1}$ whence $g$ cannot factor through $A$. This shows, by a standard $\operatorname{argument}$, that $B$ can be embedded into a product of the $Q\left(G_{i}\right)$.

3.6. injeCtive EnVelopes. By combining our results with those of Banaschewski [1970], we easily obtain,

3.7. Corollary. Under the same hypotheses, every object can be embedded into an injective envelope. 


\section{Left exact functors}

It is a familiar fact of abelian categories that a right exact functor is left exact as soon as it preserves monos. In some ways this is a very surprising fact. Despite the example of the exact sheaf reflector, no non-additive version of this has ever been stated. It is interesting that we have such a theorem for categories with effective unions. A similar theorem has been discovered by Borceux and Veit [unpublished manuscript].

4.1. Theorem. Suppose $F: \mathscr{C} \longrightarrow \mathscr{D}$ is a functor such that $\mathscr{C}$ has finite limits, cokernel pairs and effective unions and $F$ preserves finite products, regular monomorphisms and cokernel pairs. Then $F$ preserves finite limits.

In the case of an abelian category, a right exact functor automatically preserves finite products and cokernel pairs, so this implies that every right exact functor that preserves (regular) monos is left exact.

Proof. It is sufficient to show that $F$ preserves equalizers. The diagram

$$
C \longrightarrow C^{\prime} \Longrightarrow C^{\prime \prime}
$$

is an equalizer if and only if

$$
C \longrightarrow C^{\prime} \Longrightarrow C^{\prime} \times C^{\prime \prime}
$$

is and in 2 , the two arrows have a common left inverse. Since also

$$
F C \longrightarrow F C^{\prime} \longrightarrow F C^{\prime \prime}
$$

is an equalizer if and only if

$$
F C \longrightarrow F C^{\prime} \Longrightarrow F\left(C^{\prime} \times C^{\prime \prime}\right) \cong F C^{\prime} \times F C^{\prime \prime}
$$

is, it is sufficient to show that an equalizer of type 2 is preserved. Hence we can suppose without loss of generality that 1 is an equalizer of two split monos with a common left inverse. We observe that if 2 is an equalizer of that sort, then

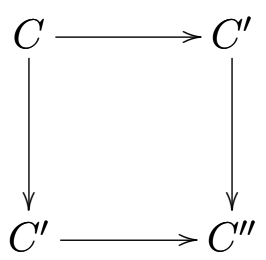

is a pullback. The arrows are all regular monos, two of them by hypothesis and the other two being split monos. It follows from the effective unions that the induced arrow $C^{\prime}+{ }_{C} C^{\prime} \longrightarrow C^{\prime \prime}$ is a regular mono. If we apply $F$ to the diagram

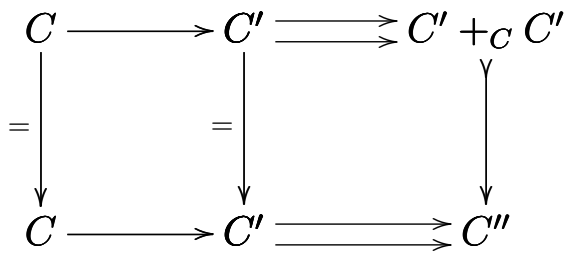


we get the diagram

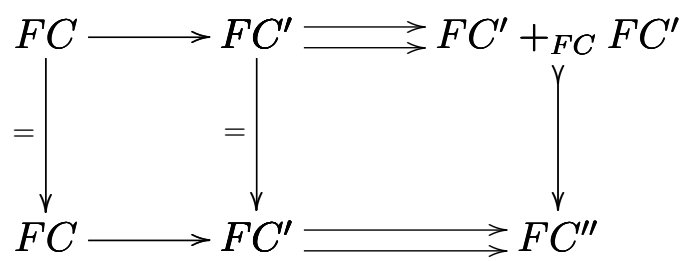

We have used the fact that $F$ preserves cokernel pairs. The arrow $F C>F C^{\prime}$ is a regular mono, hence is the equalizer of its cokernel pair. Moreover, since $F$ preserves regular monos, the induced arrow $F C^{\prime}+{ }_{F C} F C^{\prime} \longrightarrow F C^{\prime \prime}$ is also a (regular) mono. It is now a trivial diagram chase to see that the bottom line is also an equalizer.

\section{Topologies}

Not surprisingly, Theorem 4.1 can be used to give a 'soft' proof of the fact that the sheaf reflector is left exact. At that the same time, it shows that the torsion-free divisible reflector with respect to a torsion theory on an abelian category is exact. In fact, categories with effective unions and sufficient injectives seem to be the right kind of category in which to study topologies in general. We begin with a definition.

5.1. A topology, also known as a torsion theory, on a category with finite limits is a natural endomorphism of the subobject functor which is monotone, inflationary and idempotent. This means that if $\mathbf{j}: \mathrm{Sub} \longrightarrow$ Sub is the endomorphism, that with each subobject $A_{0}$ of an object $A$, there is associated a subobject $\mathbf{j} A_{0}=\mathbf{j}_{A} A_{0}$, which is

(a) (natural): If $f: B \longrightarrow A$ and $A_{0} \subseteq A$, then $f^{-1}\left(\mathbf{j}_{A} A_{0}\right)=\mathbf{j}_{B}\left(f^{-1}\left(A_{0}\right)\right)$.

(b) (monotone): If $A_{0} \subseteq A_{1} \subseteq A$, then $\mathbf{j}_{A} A_{0} \subseteq \mathbf{j}_{A} A_{1}$.

(c) (inflationary): $A_{0} \subseteq \mathbf{j} A_{0}$.

(d) (idempotent): $\mathbf{j j} A_{0}=\mathbf{j} A_{0}$.

5.2. Sheaves for a topology. Let $\mathbf{j}$ be a topology on the category $\mathscr{C}$. A subobject $C_{0} \subseteq C$ is called $\mathbf{j}$-closed if $\mathbf{j}_{C} C_{0}=C_{0}$ and $\mathbf{j}$-dense if $\mathbf{j}_{C} C_{0}=C$. An object $C$ will be called $\mathbf{j}$-separated or $\mathbf{j}$-torsion free if the diagonal $C \longrightarrow C \times C$ is $\mathbf{j}$-closed. It is called a $\mathbf{j}$-sheaf or $\mathbf{j}$-torsion free and divisible if it is $\mathbf{j}$-separated and if it is injective with respect to $\mathbf{j}$-dense monos. It is normal to omit the $\mathbf{j}$ when there is no doubt of the topology in question.

Note that we use the terminology of topologies on a category, although there is a parallel terminology developed in the context of abelian categories. 
5.3. Lemma. Let $\mathscr{C}$ be a category with a topology j. Let $A$ be an object of $\mathscr{C}$ and $B$ be a subobject of $A$. Then

(a) If

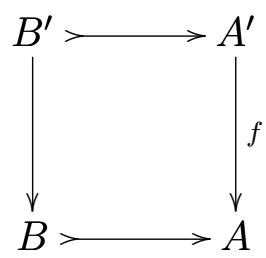

is a pullback, then so are both squares of

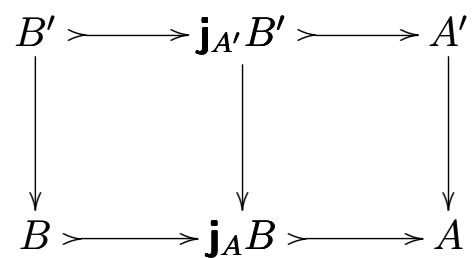

(b) If $C \subseteq B$, then $\mathbf{j}_{B} C=B \cap \mathbf{j}_{A} C$.

(c) If

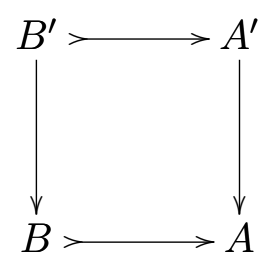

is a commutative square with the top arrow dense and the bottom arrow closed, then there is a unique arrow $A^{\prime} \longrightarrow B$ making both triangles commute.

(d) $A \subseteq \mathbf{j}_{A} B$ is dense and $\mathbf{j}_{A} B \subseteq A$ is closed; moreover these properties characterize $\mathbf{j}_{A} B$ uniquely.

(e) If $f: A^{\prime} \longrightarrow A$ is any map in $\mathscr{C}$ and $B$ is dense (respectively closed) in $A$, then $B^{\prime}=f^{-1}(B)$ is dense (respectively closed) in $B$.

(f) If $C \subseteq B \subseteq A$ and both inclusions are dense (respectively closed), then $C$ is dense (respectively closed) in $A$.

(g) If $B$ and $C$ are both dense (respectively closed) in $A$ then $B \cap C$ is dense (respectively closed) in $A$. 
ProOF. (a) That the right hand square is a pullback follows from the fact that $\mathbf{j}$ is a natural endomorphism of the subobject functor means that

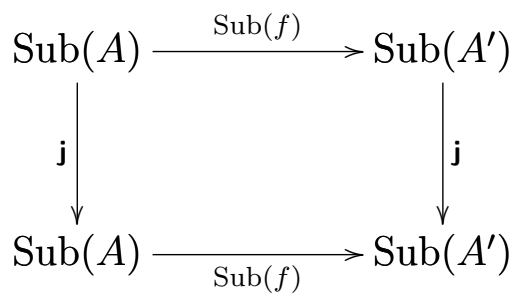

commutes. For $\operatorname{Sub}(f)(B)=B^{\prime}$ by assumption and so $\operatorname{Sub}(f)\left(\mathbf{j}_{A} B\right)=\operatorname{Sub}_{A^{\prime}} B^{\prime}$.

(b) This follows from (a) and the observation that

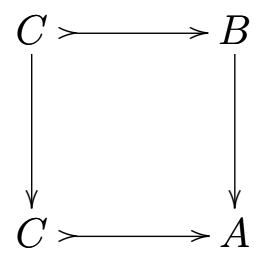

is a pullback.

(c) It follows from (a) that we have a commutative diagram

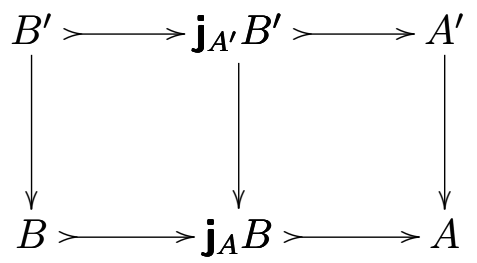

which gives existence. The uniqueness follows from the fact that $\mathbf{j}_{A} B \longrightarrow A$.

(d) $\mathbf{j}_{\mathbf{j} B} B=\mathbf{j}_{A} B \cap \mathbf{j}_{A} B=\mathbf{j}_{A} B$ from (b) which gives the density. Since $\mathbf{j}$ is idempotent, $\mathbf{j}_{A}\left(\mathbf{j}_{A} B\right)=\mathbf{j}_{A} B$, which gives the closedness. The uniqueness is assured by (c).

(e) This is immediate from (a) since a pullback of an equality is an equality.

(f) Suppose both inclusions are dense. From (b) we have that $B=\mathbf{j}_{B} C=B \cap \mathbf{j}_{A} C$ so that $B \subseteq \mathbf{j}_{A} C$, whence $A=\mathbf{j}_{A} B=\mathbf{j}_{A} \mathbf{j}_{A} C=\mathbf{j}_{A} C$. Next suppose both are closed. Then $\mathbf{j}_{A} C \subseteq \mathbf{j}_{A} B=B$. Thus $C=\mathbf{j}_{B} C=B \cap \mathbf{j}_{A} C=\mathbf{j}_{A} C$.

(g) This is immediate from (e) and (f).

5.4. Let $\mathbf{j}$ be a topology on $\mathscr{C}$. Then for $C$ an object of $\mathscr{C}$, we let $R C$ denote the j-closure of the diagonal of $C$ in $C \times C$.

5.5. Proposition. Let $\mathbf{j}$ be a topology on $\mathscr{C}$. Then for any arrows $f, g: A \longrightarrow C$, the equalizer of $f$ and $g$ is dense in $A$ if and only if $\langle f, g\rangle: A \longrightarrow C \times C$ factors through $R C$. 
Proof. The equalizer $E$ of $f$ and $g$ is characterized by the fact that

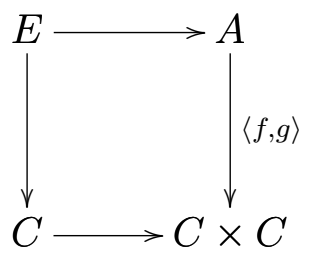

is a pullback. Applying $\mathbf{j}$, we get

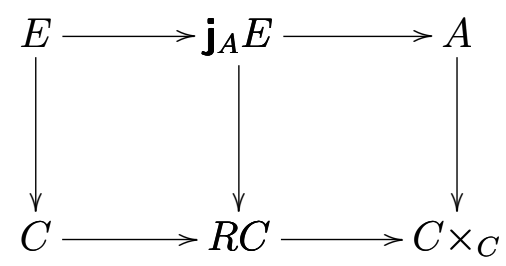

If $E$ is dense, we see the required factorization immediately. If $\langle f, g\rangle$ factors through $R C$, the fact that the right hand square is a pullback implies that $\mathbf{j}_{A} E=A$ and then $E$ is dense.

5.6. Corollary. Two maps to a separated object that agree on a dense subobject are equal.

5.7. Proposition. Suppose $\mathbf{j}$ is a topology on a category $\mathscr{C}$. Then for any object $C$, $R C$ is an equivalence relation on $C$.

Proof. Since the intersection of dense subobjects is dense, one easily shows that the relation on $\operatorname{Hom}(A, C)$ of agreeing on a dense subobject of $C$ is an equivalence relation and is the relation of factoring through $R C$.

5.8. We will call a topology $\mathbf{j}$ on $\mathscr{C}$ effective if for each object $C$ of $\mathscr{C}, R C$ is an effective equivalence relation on $C$.

5.9. Proposition. Let $\mathbf{j}$ be an effective topology on the regular category $\mathscr{C}$ and $S C=C / R C$ denote the quotient functor. Then for any object $C, S C$ is separated and is the separated reflection of $C$.

Proof. In the pullback diagram

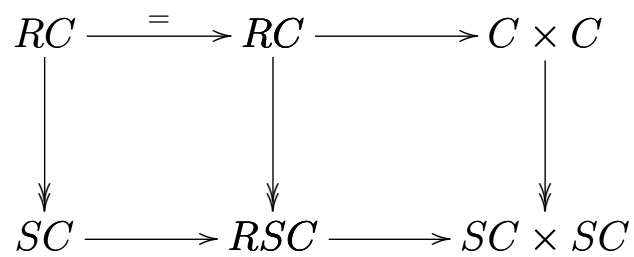

the fact that $R C \longrightarrow R S C$ is a regular epi implies that $S C \longrightarrow R S C$ is as well and hence, being a mono, is an isomorphism. 
This shows that $S C$ is separated. If $A$ is separated and $f: C \longrightarrow A$ is an arrow, we have

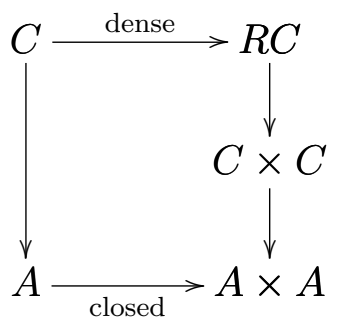

whose diagonal fill-in shows that the arrow $C \longrightarrow A$ coequalizes the kernel pair and hence that there is an induced map $S C \longrightarrow A$. The uniqueness is evident.

5.10. Proposition. Under the same hypotheses, $S$ preserves monos and finite products.

Proof. It follows from Lemma 5.3(e) that $R A \times B$ and $A \times R B$ are dense in $R A \times R B$ and hence by part (g) that their intersection $A \times B$ is dense in $R A \times R B$. It similarly follows that $R A \times R B$ is closed in $A \times A \times B \times B$ and hence by (d) that the $\mathbf{j}$-closure of $A \times B$ is $R A \times R B$, in other words that $R(A \times B)=R A \times R B$. In a regular category, a product of coequalizers is a coequalizer so that

$$
R A \times R B \longrightarrow A \times B \longrightarrow S A \times S B
$$

is a coequalizer and hence $S(A \times B) \cong S A \times S B$.

As for monos, if (and only if) $A \longrightarrow B$ is monic,

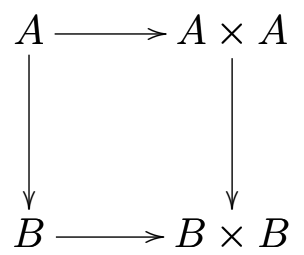

is a pullback and hence so is

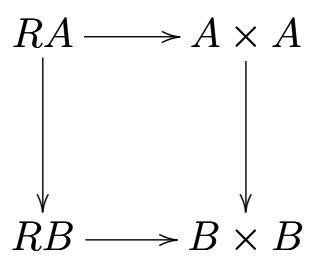

by Lemma 5.3(a). It is then a simple exercise, using the regular embedding of [Barr, 1971] or [Barr, 1986] to see that the induced $S A \longrightarrow S B$ is monic.

5.11. Proposition. Suppose that $\mathscr{C}$ is regular, that pushouts of monos exist and are mono and that $\mathbf{j}$ is an effective topology on $\mathscr{C}$. Then an object $F$ is a $\mathbf{j}$-sheaf if and only if $F$ is separated and is not $\mathbf{j}$-dense in any properly containing separated object. 
Proof. If $F$ is a sheaf, then let $f: F \longrightarrow C$ be a j-dense monic. The injectivity of $F$ with respect to $\mathbf{j}$-dense monics implies the existence of a map $g: B \longrightarrow F$ such that $g \circ f$ is the identity. Then $f \circ g$ and the identity of $C$ agree when composed with the $\mathbf{j}$-dense monic $f$ and so are equal. To go the other way, suppose $F$ has no proper separable dense extensions. Then supposing we have

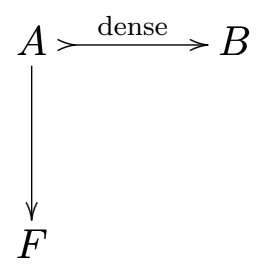

we form the pushout:

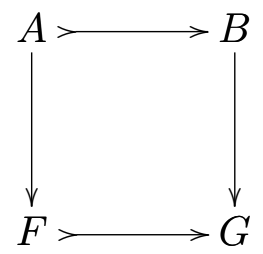

With $F$ closed in $G$, Lemma 5.3(c) gives the required map.

5.12. Theorem. Suppose that $\mathscr{C}$ is regular with every mono regular and that $\mathbf{j}$ is an effective topology on $\mathscr{C}$. Suppose each object can be embedded in an injective. Then each separated object is embedded in a sheaf.

Proof. Let $C$ be separated and $f: C \longrightarrow Q$ an injective container. Then $f$ induces a mono $S f: S C \longrightarrow S Q$. Since $C$ is separated, $C=S C$ and we have $S f=g \circ f: C$ $\longrightarrow S Q$ where $g: Q \longrightarrow S Q$ is the canonical map. A subobject of a separated object is separated (an easy consequence of Lemma 5.3(e)) so that if we factor $S f=r \circ m$, where $m: C>F$ is dense and $r: F>S Q$ is closed, then $F$ is separated. Now we claim that $F$ is a sheaf. In fact, let $h: A \longrightarrow B$ be a dense mono and $k: A \longrightarrow F$ an arrow. Define $A_{0}$ so that the upper left square in the diagram

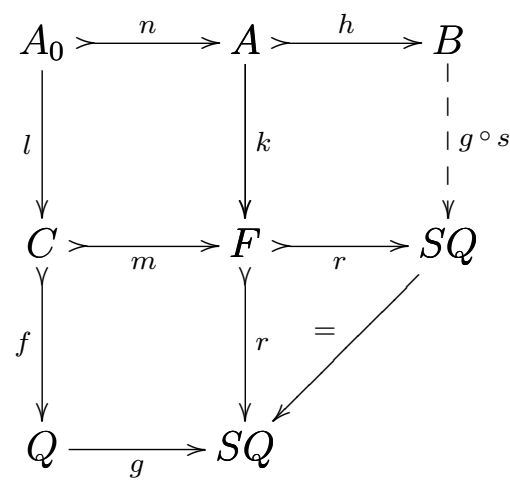

is a pullback. Now the injectivity of $Q$ implies the existence of a map $s: B \longrightarrow Q$ such that $t \circ h \circ n=f \circ l$. Then

$$
g \circ s \circ h \circ n=g \circ f \circ l=r \circ m \circ l=r \circ k \circ n \circ
$$


But the target, $S Q$, of that map is separated and $n$ is dense so that $g \circ s \circ h=r \circ k$ and the right hand square commutes. Finally, $m$ is assumed dense and $s$ is closed by the definition of $F$ and so 5.3(c) gives the desired conclusion.

5.13. THEOREM. Under the same hypotheses, a subobject of a sheaf is separated and its closure is a sheaf, in fact, the sheaf reflector of that object.

Proof. Any subobject of a sheaf (or of any separated object), is readily seen to be separated. Let $A$ be such an object and let $A \longrightarrow F$ be dense with $F$ a sheaf. Consider a diagram

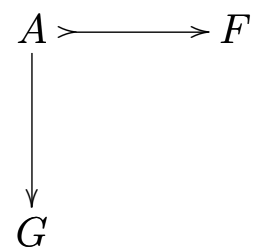

with $G$ a sheaf. Since $G$ is a sheaf and the arrow $A \longrightarrow F$ is dense, it follows there is a map $F \longrightarrow G$ making the triangle commute. It is unique because two maps to a separated object that agree on a dense subobject are equal.

5.14. TheOREM. Suppose that $\mathscr{C}$ is a category and $\mathbf{j}$ a topology on $\mathscr{C}$ that satisfy the hypotheses of Theorem 5.12. Then the inclusion of the full category of $\mathbf{j}$-sheaves has a left adjoint that preserves monos and finite products.

Proof. Only the preservation properties need be verified. The preservation of monos is easy since if $A \longrightarrow B$, we have $S A \longrightarrow S B$ and if $F B$ is the sheaf associated to $S B$, the sheaf associated to $A$ is the $\mathbf{j}$-closure of $S A$ under the inclusion $S A>S B \longrightarrow F B$. As for products, the product (including empty product) of sheaves is a sheaf because the inclusion is a right adjoint. Then $S(A \times B) \cong S A \times S B>F A \times F B$ is dense from Lemma 5.3(e) and (g).

5.15. Corollary. If, in addition, $\mathscr{C}$ has effective unions, then the associated sheaf functor is left exact.

5.16. THEOREM. Suppose the hypotheses of the preceding corollary are satisfied. Then the category $\mathscr{C}_{\mathbf{j}}$ of $\mathbf{j}$-sheaves is regular with all monos regular and effective unions. If pushouts of monos are mono in $\mathscr{C}$, they are in $\mathscr{C}_{\mathbf{j}}$; if $\mathscr{C}$ has effective equivalence relations, so does $\mathscr{C}_{\mathbf{j}}$.

ProOF. Being a reflective subcategory of a category with finite limits, $\mathscr{C}_{\mathbf{j}}$ has them too. If

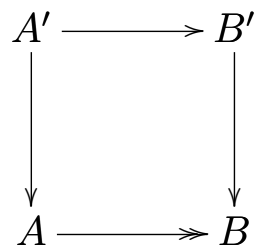


is a pullback in the category of sheaves, it is in $\mathscr{C}$ since the inclusion is a right adjoint. Then the arrow $A^{\prime} \longrightarrow B^{\prime}$ is a regular epi in $\mathscr{C}$, a fortiori in $\mathscr{C}_{\text {j }}$.

A mono in $\mathscr{C}_{\mathbf{j}}$ is regular in $\mathscr{C}$ and by applying the associated sheaf functor to the corresponding equalizer diagram, we conclude that it is regular in $\mathscr{C}_{\mathbf{j}}$ as well.

To see that unions are effective, we consider a pullback of monos in $\mathscr{C}_{\mathbf{j}}$.

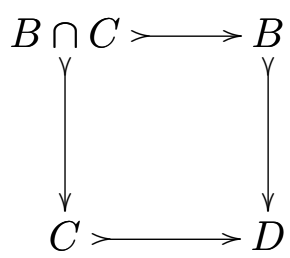

This is also an intersection in $\mathscr{C}$. If $D^{\prime} \longrightarrow D$ is the pushout in $\mathscr{C}$, then $F D^{\prime}>F D=$ $D$ is the pushout in $\mathscr{C}_{\mathbf{j}}$. The universal mapping property of the adjoint insures that any subsheaf of $D$ that includes both $B$ and $C$ also includes $F D^{\prime}$, so it is also their union in the subobject lattice.

Next, consider a pushout in $\mathscr{C}$

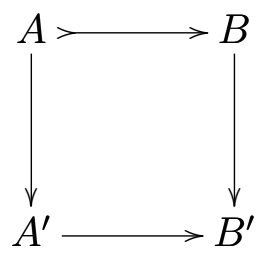

in which $A, B$ and $A^{\prime}$ are sheaves and the upper arrow is monic. Since left adjoints preserve pushouts, the pushout in $\mathscr{C}_{\mathbf{j}}$ is the sheaf associated to $B^{\prime}$. Since the reflector preserves monos, the arrow $A^{\prime} \longrightarrow F B$ is still monic.

Suppose that equivalence relations in $\mathscr{C}$ are effective. Let $A$ be a sheaf and $E \subseteq A \times A$ an equivalence relation which is also a sheaf. Then we have a kernel pair diagram in $\mathscr{C}$

$$
E \Longrightarrow A \longrightarrow B
$$

Applying the associated sheaf functor $F$, we get that

$$
E \longrightarrow A \longrightarrow F B
$$

is also a kernel pair since the associated sheaf functor preservers coequalizers. The conclusion now follows since we have shown that all monos in $\mathscr{C}_{\mathbf{j}}$ are regular.

\section{References}

B. Banaschewski, Injectivity and essential extensions in equational classes of algebras. Proc. Conf.. on Universal Algebra, (1969). Queen's Series Pure Applied Math., 25 (1970). 
M. Barr, Exact categories. In Exact Categories and Categories of Sheaves, Springer Lecture Notes in Mathematics 236 (1971), 1-120.

M. Barr, Non-abelian torsion theories. Canad. J. Math., 25 (1973), 1224-1237.

M. Barr, Representations of categories. J. Pure and Applied Algebra, 41 (1986), 113-137.

F. Borceux \& B. Veit, On the left exactness of orthogonal reflections. Unpublished manuscript.

D. Buchsbaum, Exact categories. Appendix to H. Cartan \& S. Eilenberg, Homological Algebra, Princeton University Press, Princeton, N. J., 1956.

A. Grothendieck, Sur quelques points d'algèbre homologique. Tohôku Math. Journal 2 (1957), 199-221.

P. M. Johnstone, Topos Theory. Cambridge University Press, 1977.

S. Mac Lane, Duality for groups. Bull. Amer. Math. Soc. 56 (1950), 485-516.

Department of Mathematics and Statistics, McGill University

805 Sherbrooke St. W Montreal, Quebec Canada H3A 2 K6

Email: barr@barrs.org 